\author{
Nicola Morelli \\ Simone Gallerini \\ Alberto Chiti \\ Mirco Cosottini \\ Paolo De Simone \\ Giovanni Orlandi \\ Luigi Murri
}

\section{Spinal MRI and intracranial hypotension diagnosis}

Received: 30 August 2003

Accepted in revised form: 6 September 2006

Published online: 25 October 2006
We thank Dr. Albayram for his interest in our case report [1]. Dr. Albayram correctly points out that CT myelography with iodinated contrast medium is the gold standard in the diagnostic and management algorithm for the intracranial hypotension syndrome.

Unfortunately, CT myelography is not routinely performed in most centres, as it entails administration of iodinated contrast media in the subarachnoid space by means of lumbar puncture [2] The potential for cerebral herniation or infection due to this manoeuvre, although theoretical, often suggests that alternative and less invasive investigations be undertaken in patients with intracranial hypotension to detect the site of the cerebral spinal fluid (CSF) leak. Until recently, spinal MRI has played little role in the diagnosis of spontaneous intracranial hypotension, as it appeared less sensitive in localising the CSF leak site. However, several spinal manifestations of spontaneous intracranial hypotension have been described over the past few years, such as the presence of dural enhancement, dilated epidural veins, extrathecal CSF collections and meningeal diverticula [3]. Therefore, spinal MRI has emerged as an elective, non-invasive investigation to search for direct or indirect signs of CSF leaks [4]. These findings, together with the patient's clinical history and symptomatology, may allow for diagnosis of the intracranial hypotensive syndrome. Based on our experience, if conservative measures, such as bed rest, oral hydration, generous caffeine intake and use of abdominal bandage prove beneficial for symptoms and radiologic findings, more invasive manoeuvres can be avoided. Should these measures be ineffective, accurate identification of the CSF leak site may be necessary with more invasive investigations - i.e., CT myelography or radionuclide cisternography - to plan eventual surgical treatment.

Leaking meningeal diverticula can be ligated, and other defects can be suture closed or repaired with the aid of pledgets, gelfoam and fibrin sealants. Use of epidural blood patches has also been described in such cases.

As Dr. Albayram observed, Figure 2 actually illustrates a non-contrast, T2weighted axial myelographic sequence. We deeply apologise for the mistake. However, the presence of longer dural sleeve surrounding a spinal nerve root was indicative that the meningeal diverticula reported in Figure 2 could be related to the site of a CSF dripping [5].

N. Morelli (西), S. Gallerini, A. Chiti, M. Cosottini, P. De Simone, G. Orlandi, L. Murri University Centre for Adaptive Disorders and Headache (UCADH), Institute of Neurology, Department of Neurosciences, University of Pisa,

Via Roma 67, I-56126, Pisa, Italy e-mail:n.morelli@inwind.it 


\section{References}

1. Morelli N, Gallerini S, Gori S et al (2006) Intracranial hypotension syndrome following chiropractic manipulation of the cervical spine. J Headache Pain 7:211-213

2. Chin CT (2002) Spine imaging. Semin Neurol 22:205-220
3. Schievink WI (2006) Spontaneous spinal cerebrospinal fluid leaks and intracranial hypotension. JAMA 295:2286-2296

4. Weindling SM, Kotsenas AL (2005) Spontaneous craniospinal hypotension. J Magn Reson Imaging 22:804-809
5. Inamasu J, Guiot BH (2006)

Intracranial hypotension with spinal pathology. Spine J 6:591-599 